\title{
Heavy-quark spin-symmetry partners of hadronic molecules
}

\section{Baru*}

Institut für Theoretische Physik II, Ruhr-Universität Bochum, D-44780 Bochum, Germany and Institute for Theoretical and Experimental Physics, B. Cheremushkinskaya 25, Moscow, Russia

E-mail: vadimbetp2.rub.de

\section{E. Epelbaum}

Institut für Theoretische Physik II, Ruhr-Universität Bochum D-44780 Bochum, Germany

E-mail: evgeny.epelbaumeruhr-uni-bochum.de

\section{A. A. Filin}

Institut für Theoretische Physik II, Ruhr-Universität Bochum D-44780 Bochum, Germany

E-mail: arseniy.filinetp2.ruhr-uni-bochum.de

\section{Hanhart}

Institute for Advanced Simulation, Institut für Kernphysik and Jülich Center for Hadron Physics, Forschungszentrum Jülich, D-52425 Jülich, Germany

E-mail: c.hanhartefz-juelich.de

\section{A. Nefediev}

Institute for Theoretical and Experimental Physics, B. Cheremushkinskaya 25, Moscow, Russia, National Research Nuclear University MEPhI, Kashirskoe highway 31, Moscow, Russia and Moscow Institute of Physics and Technology, Institutsky lane 9, Dolgoprudny, Moscow Region, Russia

E-mail: nefediev@itep.ru

Implications of heavy-quark spin-symmetry are investigated for the isoscalar charmonium-like state $X(3872)$ and the isovector bottomonium-like states $Z_{b}(10610)$ and $Z_{b}(10650)$ under the assumption of the latter being bound states of the nearby heavy meson-antimeson pairs. We formulate and solve a system of the integral equations for a coupled-channel problem involving the $P \bar{P}, P \bar{V}$, and $V \bar{V}$ channels (with $P$ and $V$ being either $D$ and $D^{*}$ or $B$ and $B^{*}$ mesons) to determine the scattering amplitudes in the channels with the quantum numbers $J^{P C}=1^{++}, 1^{+-}, 0^{++}$, and $2^{++}$. The coupled-channel potentials incorporate the contact and one-pion exchange interactions derived in a chiral effective field theory approach and iterated to all orders. Once two contact terms at leading order are adjusted to reproduce the binding energies of the states used as input, the approach can be employed to predict the mass and the prominent contributions to the width of the spin-partner states with the quantum numbers $J^{++}(J=0,1,2)$.

XVII International Conference on Hadron Spectroscopy and Structure - Hadron2017

25-29 September, 2017

University of Salamanca, Salamanca, Spain

\footnotetext{
*Speaker.
} 


\section{Introduction}

Chiral EFT is a well established approach to probe QCD in the non perturbative regime. It is organised as an expansion with respect to the ratio of soft dynamical scales to the large scale where chiral symmetry breaks down. The chiral potential at each given order can be written as a sum of dynamical contributions (such as one-pion exchange potential) and a series of contact terms which can not be fixed from chiral symmetry. Chiral EFT has already revealed itself as a very useful tool for studies of various processes not only in meson and one-baryon sectors but also for investigating shallow states of several nucleons, see, for example, reviews [1, 2, 3].

Recently, several quarkonium-like states lying very close to some hadronic thresholds have been experimentally observed, for reviews see, e.g., Refs. [4, 5, 6, 7] . The most prominent examples are the $\mathrm{X}(3872)$ residing near the $D \bar{D}^{*}$ threshold and the two bottomonium-like states $Z_{b}(10610)$ and $Z_{b}(10650)$ located in the vicinity to the $B \bar{B}^{*}$ and $B^{*} \bar{B}^{*}$ thresholds, respectively. It is crucial that these states couple to the corresponding nearby hadronic channels in S-waves and exhibit large branching fractions to these open-flavour channels $[8,9,10]$. Therefore, these shallow states, being similar to the deuteron in nuclear physics, provide a very appealing possibility to test predictions of chiral EFT in the quarkonium sector. Moreover, in addition to chiral symmetry applied to the light quarks, shallow quarkonium states obey heavy-quark symmetries, which make it possible to predict new exotic states - the spin partners of the $\mathrm{X}(3872)$ and the $Z_{b}$ 's. In this contribution, we review the main findings of Refs. [11, 12], where the spin partner states of the $\mathrm{X}(3872)$ and the $Z_{b}$ 's were predicted using the chiral EFT framework with nonperturbative pions. In addition, the impact of scales related to three-body unitarity on the location of the $J^{P C}=2^{++}$ (tensor) spin partner of the $\mathrm{X}(3872)$ is discussed for the first time.

\section{Chiral EFT based approach for hadronic molecules}

A complete system of basis vectors for the quantum numbers $J^{P C}=1^{++}, 1^{+-}, 0^{++}$, and $2^{++}$ composed of pseudoscalar $(P=D$ or $B)$ and/or vector $\left(V=D^{*}\right.$ or $\left.B^{*}\right)$ mesons reads

$$
\begin{aligned}
0^{++}: & \left\{P \bar{P}\left({ }^{1} S_{0}\right), V \bar{V}\left({ }^{1} S_{0}\right), V \bar{V}\left({ }^{5} D_{0}\right)\right\}, \\
1^{+-}: & \left\{P \bar{V}\left({ }^{3} S_{1},-\right), P \bar{V}\left({ }^{3} D_{1},-\right), V \bar{V}\left({ }^{3} S_{1}\right), V \bar{V}\left({ }^{3} D_{1}\right)\right\}, \\
1^{++}: & \left\{P \bar{V}\left({ }^{3} S_{1},+\right), P \bar{V}\left({ }^{3} D_{1},+\right), V \bar{V}\left({ }^{5} D_{1}\right)\right\}, \\
2^{++}: & \left\{P \bar{P}\left({ }^{1} D_{2}\right), P \bar{V}\left({ }^{3} D_{2}\right), V \bar{V}\left({ }^{5} S_{2}\right), V \bar{V}\left({ }^{1} D_{2}\right), V \bar{V}\left({ }^{5} D_{2}\right), V \bar{V}\left({ }^{5} G_{2}\right)\right\} .
\end{aligned}
$$

where $P \bar{V}( \pm)=\frac{1}{\sqrt{2}}(P \bar{V} \pm V \bar{P})$ label C-parity even and odd states, respectively, and the partial waves of the corresponding heavy meson pairs are shown in parentheses. The potentials at leading order (LO) connecting these basis vectors consist of two S-wave contact interactions, one-pion (OPE) as well as one-eta (OEE) interactions iterated to all orders via the nonperturbative solutions of the integral equations of the Lippmann-Schwinger type. Due to constraints from the heavy-quark spin symmetry, at leading order in the isospin limit there exist only two contact terms contributing to $S$ waves [13, 14, 15], that is 


$$
\begin{aligned}
& V_{\mathrm{LO}}^{(0++)}=\frac{1}{4}\left(\begin{array}{cc}
3 C_{I}+C_{I}^{\prime} & -\sqrt{3}\left(C_{I}-C_{I}^{\prime}\right) \\
-\sqrt{3}\left(C_{I}-C_{I}^{\prime}\right) & C_{I}+3 C_{I}^{\prime}
\end{array}\right), \quad V_{\mathrm{LO}}^{(1+-)}=\frac{1}{2}\left(\begin{array}{c}
C_{I}+C_{I}^{\prime} C_{I}-C_{I}^{\prime} \\
C_{I}-C_{I}^{\prime} C_{I}+C_{I}^{\prime}
\end{array}\right), \\
& V_{\mathrm{LO}}^{(1++)}=V_{\mathrm{LO}}^{(2++)}=C_{I},
\end{aligned}
$$

where $\left\{C_{I}, C_{I}^{\prime}\right\}$ are the low-energy constants (LECs) corresponding to a definite isospin (I), and the other, non-S-wave, transitions were omitted. Then, the OPE potentials (before partial-wave projection) connecting various heavy-meson pairs in the initial and final state read ${ }^{1}$

$$
\begin{aligned}
& V_{P \bar{V} \rightarrow V \bar{P}}\left(\boldsymbol{p}, \boldsymbol{p}^{\prime}\right)=-\frac{2 g^{2}}{\left(4 \pi f_{\pi}\right)^{2}}\left(\boldsymbol{\tau}_{1} \cdot \boldsymbol{\tau}_{2}^{c}\right)\left(\boldsymbol{\varepsilon}_{1} \cdot \boldsymbol{q}\right)\left(\boldsymbol{\varepsilon}_{2}^{\prime *} \cdot \boldsymbol{q}\right)\left(\frac{1}{D_{P P \pi}\left(\boldsymbol{p}, \boldsymbol{p}^{\prime}\right)}+\frac{1}{D_{V V \pi}\left(\boldsymbol{p}, \boldsymbol{p}^{\prime}\right)}\right), \\
& V_{P \bar{V} \rightarrow V \bar{V}}\left(\boldsymbol{p}, \boldsymbol{p}^{\prime}\right)=\frac{2 \sqrt{2} g^{2}}{\left(4 \pi f_{\pi}\right)^{2}}\left(\tau_{1} \cdot \boldsymbol{\tau}_{2}^{c}\right)\left(\boldsymbol{A}_{1} \cdot \boldsymbol{q}\right)\left(\boldsymbol{\varepsilon}_{2}^{\prime *} \cdot \boldsymbol{q}\right)\left(\frac{1}{D_{P V \pi}\left(\boldsymbol{p}, \boldsymbol{p}^{\prime}\right)}+\frac{1}{D_{V V \pi}\left(\boldsymbol{p}, \boldsymbol{p}^{\prime}\right)}\right), \\
& V_{P \bar{P} \rightarrow V \bar{V}}\left(\boldsymbol{p}, \boldsymbol{p}^{\prime}\right)=-\frac{2 g^{2}}{\left(4 \pi f_{\pi}\right)^{2}}\left(\boldsymbol{\tau}_{1} \cdot \boldsymbol{\tau}_{2}^{c}\right)\left(\boldsymbol{\varepsilon}_{1}^{\prime *} \cdot \boldsymbol{q}\right)\left(\boldsymbol{\varepsilon}_{2}^{\prime *} \cdot \boldsymbol{q}\right)\left(\frac{2}{D_{P V \pi}\left(\boldsymbol{p}, \boldsymbol{p}^{\prime}\right)}\right), \\
& V_{V \bar{V} \rightarrow V \bar{V}}\left(\boldsymbol{p}, \boldsymbol{p}^{\prime}\right)=-\frac{4 g^{2}}{\left(4 \pi f_{\pi}\right)^{2}}\left(\boldsymbol{\tau}_{1} \cdot \boldsymbol{\tau}_{2}^{c}\right)\left(\boldsymbol{A}_{1} \cdot \boldsymbol{q}\right)\left(\boldsymbol{A}_{2} \cdot \boldsymbol{q}\right)\left(\frac{2}{D_{V V \pi}\left(\boldsymbol{p}, \boldsymbol{p}^{\prime}\right)}\right),
\end{aligned}
$$

where $\boldsymbol{\varepsilon}$ and $\boldsymbol{\varepsilon}^{\prime *}$ stand for the polarisation vectors of the initial and final $V$ mesons, respectively, $\boldsymbol{A}=\frac{i}{\sqrt{2}}\left(\boldsymbol{\varepsilon} \times \boldsymbol{\varepsilon}^{\prime *}\right), \boldsymbol{p}\left(\boldsymbol{p}^{\prime}\right)$ denotes the centre-of-mass momentum of the initial (final) heavy-meson pair, $\boldsymbol{q}=\boldsymbol{p}+\boldsymbol{p}^{\prime}$ stands for the pion momentum, and $f_{\pi}=92.2 \mathrm{MeV}$ is the pion decay constant. The isospin factor in Eqs. (2.4) which appears from the operator $\tau_{1} \cdot \tau_{2}^{c}$ is

$$
\tau_{1} \cdot \tau_{2}^{c}=[3-2 I(I+1)],
$$

which gives -1 for the isovector channels and +3 for isoscalars. Furthermore, the pion coupling to the heavy mesons, $g \approx 0.57$, was extracted from the observable $D^{*} \rightarrow D \pi$ decay width. Assuming heavy-flavour symmetry, the same value is also used in the B-sector. This is also supported by the recent lattice QCD determination of the $B B^{*} \pi$ coupling constant $\left(g_{b}\right)$ [16] consistent with the aforementioned value of $g$. The time-ordered three-body propagators in Eqs. (2.4) read

$$
D_{H_{1} H_{2} \pi}\left(\boldsymbol{p}, \boldsymbol{p}^{\prime}\right)=2 E_{\pi}(\boldsymbol{q})\left(E_{H_{1}}(p)+E_{H_{2}}\left(p^{\prime}\right)+E_{\pi}(\boldsymbol{q})-\sqrt{s}-i \varepsilon\right),
$$

where $E_{\pi}(\boldsymbol{q})=\sqrt{\boldsymbol{q}^{2}+m_{\pi}^{2}}, E_{H_{1(2)}}(p)=\sqrt{p^{2}+m_{H_{1(2)}}^{2}}$, with $m_{\pi}$ and $m_{H_{1(2)}}$ standing for the masses of the pion and the heavy mesons (either $P$ or $V$ mesons in Eq. (2.4)), respectively, and $\sqrt{s}$ is the energy of the system. Since the $D \bar{D} \pi$ threshold is located below the $D \bar{D}^{*}$ one $e^{2}$, the potentials involving the propagator $D_{D D \pi}$ possess a three-body cut in the energy regime where the $\mathrm{X}(3872)$ resides. Another source of this cut is the self energy of the $D^{*}$ meson incorporated in the $D \bar{D}^{*}$ Green function in the Lippmann-Schwinger-type equations, see Refs. [17, 18, 19] for more details. Although the largest dynamical effect from OPE on the partner states is associated with much larger scales (the momenta $\simeq \sqrt{m_{H}\left(m_{V}-m_{P}\right)}$ ) from coupled-channel effects, the inclusion of the ultra-soft scales from the three-body cut also has an impact on the location of the spin partners of the $X(3872)$, as discussed in the next section.

\footnotetext{
${ }^{1}$ The expressions for the OEE potentials are similar and can be found, e.g., in Ref. [12].

${ }^{2}$ We work in the isospin limit and use average masses of the heavy mesons and the pions.
} 


\section{Results}

In order to make predictions based on the chiral EFT approach formulated in the previous section, first the LECs have to be pinned down. In the charmonium sector, we use as input the $X(3872)$ treated as an isoscalar shallow bound $D \bar{D}^{*}$ state. By solving the system of the integral equations in the $1^{++}$channel we adjust the LEC $C_{0}$ to reproduce the binding energy of the $X(3872)$. Unfortunately, no other unambiguous isoscalar candidates for the shallow molecular states have been identified in the experimental spectrum so far. Therefore, at present predictions are possible for the $2^{++}$state only, where the contact interaction is described by exactly the same LEC $C_{0}$ (see Eq. (2.3)). On the contrary, in the bottomonium sector, the experimental discovery of the two nearthreshold isovector resonances, $Z_{b}$ and $Z_{b}^{\prime}$, allows one to tune both contact terms, $C_{1}$ and $C_{1}^{\prime}$, and therefore to predict all partner states in this multiplet.

If pions are integrated out, the partner states both in the charmonium and bottomonium spectra reside near the corresponding sibling thresholds. For example, the $2^{++}$partner in the $c$ - and $b$-sector are located close to the respective $V \bar{V}$ threshold. The main impact of the pions reveals itself in additional coupled-channel transitions between various heavy-meson states which otherwise (i.e. without pions) would be strongly suppressed. Since these transitions turned out to proceed predominantly in D-waves, as can be seen from the basis vectors in Table 1, the most important impact on the observables is provided by the tensor forces driven by OPE. In order to illustrate the impact of OPE on the partner states of the $Z_{b}$ 's, in Fig.1 we show the dependence of the binding energies in the $0^{++}$and $2^{++}$channels (defined relative to the nearby thresholds) on the pionic coupling constant $g_{b}$ varied from zero to the physical value. Note that for each value of $g_{b}$ the contact terms were refitted to reproduce the input values for the $Z_{b}$ 's binding energies. It is clear that the contribution from the central (S-wave) part of OPE can be fully absorbed into the redefinition of the LECs, while the one from the tensor forces provides a nontrivial dynamical effect for the physical value of $g_{b}$.
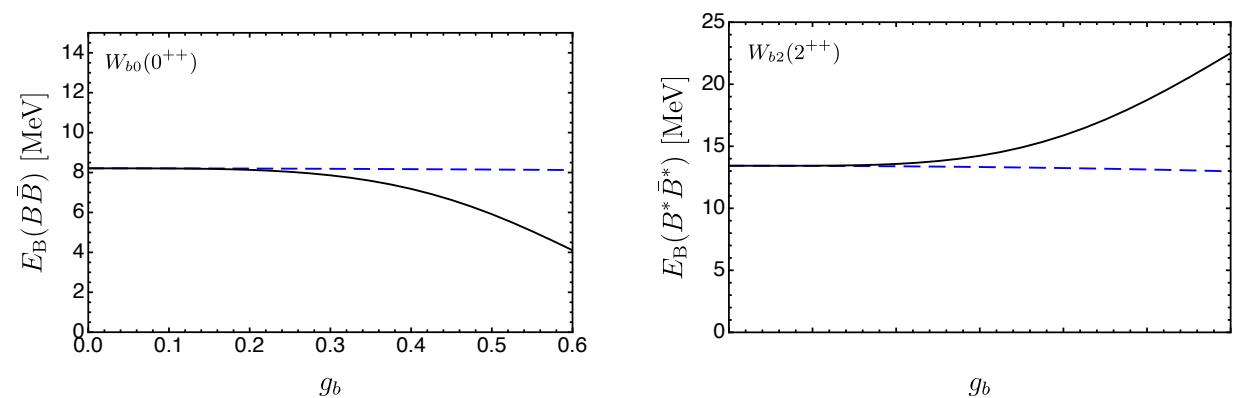

Figure 1: Evolution of the binding energies of the $Z_{b}$ 's spin partners as functions of the pionic coupling constant with heavy mesons. The blue dashed curves are obtained for the problem with the contact plus central ( $S$-wave) part of the OPE interactions; the black solid curves represent the full results, including tensor forces from OPE. The left edge of the plot corresponds to the case of the pionless (purely contact) theory, while the right edge shows the results for the physical value of $g_{b}$.

In Fig. 2 we compare the impact of nonperturbative pion dynamics on the tensor spin-partner states in the $c$-and $b$-quark sectors. Similarly to the $b$-quark sector, the leading non-trivial effect from the OPE on the charmonium-like states originates from the $(S-D)$ coupled-channel transitions 
that implies that a perturbative inclusion of the OPE is not sufficient. In the bottom sector, we predict the existence of a narrow $2^{++}$tensor state $W_{b 2}$, with a width of the order of several $\mathrm{MeV}$, lying a few $\mathrm{MeV}$ below the $B^{*} \bar{B}^{*}$ threshold. On the contrary, the corresponding tensor spin partner of the $X(3872)$ is predicted to have a much larger width as well as to be stronger shifted away from the $D^{*} \bar{D}^{*}$ threshold because of a stronger HQSS violation in the charmonium case caused by nonperturbative pion exchanges. The role of the three-body dynamics (from the intermediate states involving one pion and two heavy mesons) turns out to be also quite different: while three-body effects are very marginal in the $b$-sector, they provide a sizeable (up to 20\%) reduction of both the partner-state binding energy and its width in the $c$-sector. Finally, an analysis of the theoretical uncertainties in the predictions for the $X_{c 2}$ and $W_{b 2}$ reveals that they originate from very different sources. Indeed, while the main source of the theoretical uncertainty for the $X_{c 2}$ is associated with the UV regulator dependence in the integral equations (which might entail the importance of higher order corrections), the predictions for the $W_{b 2}$ are basically independent of the regulator but show some sensitivity to the input for the binding energies of the $Z_{b}$ 's states.

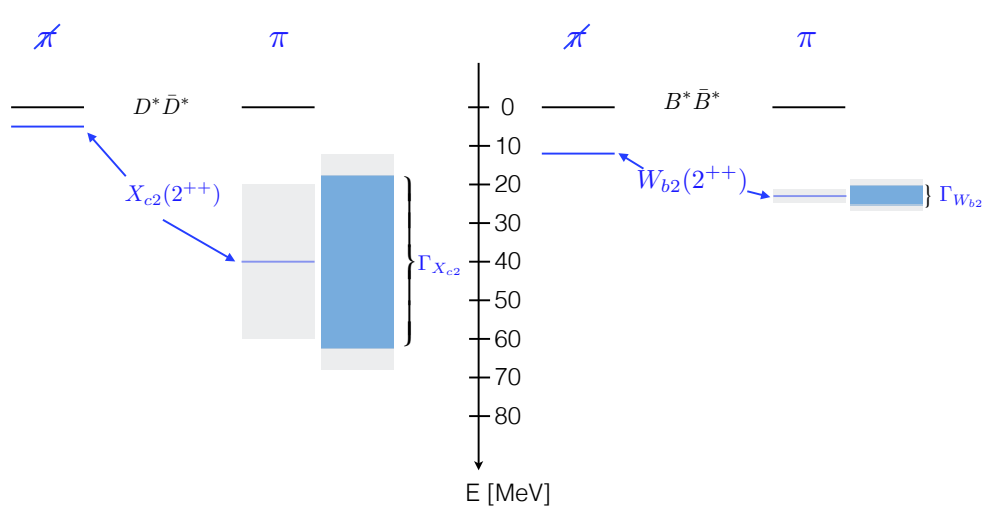

Figure 2: Tensor spin partners of the $X(3872)$ (left panel) and the $Z_{b}$ states (right panel) predicted in the contact $(\pi)$ and full $(\pi)$ theory. Blue horizontal lines indicate the energies of the states relative to the $D^{*} \bar{D}^{*}$ and $B^{*} \bar{B}^{*}$ thresholds, respectively. Blue box corresponds to the prominent contribution to the width of the corresponding state from the decays to open-flavour channels predicted in the pionful theory. Grey box represents the theoretical uncertainty.

To summarise, in this Contribution we discussed a way to formulate a chiral EFT approach to study various hadronic molecules and summarised the implications of this approach for the spin partners of the $\mathrm{X}(3872)$ and the two bottomonium-like states $Z_{b}(10610)$ and $Z_{b}(10650)$ with the special emphasis on the tensor spin-partner states.

This work is supported in part by the DFG and the NSFC through funds provided to the Sino-German CRC 110 "Symmetries and the Emergence of Structure in QCD" (NSFC Grant No. 11261130311). Work of A. N. was performed within the Institute of Nuclear Physics and Engineering supported by MEPhI Academic Excellence Project (contract No 02.a03.21.0005, 27.08.2013). He also acknowledges support from the Russian Foundation for Basic Research (Grant No. 17-0200485). Work of V. B. is supported by the DFG (Grant No. GZ: BA 5443/1-1). 


\section{References}

[1] V. Bernard, N. Kaiser and U.-G. Meißner, Int. J. Mod. Phys. E 4, 193 (1995).

[2] V. Bernard, Prog. Part. Nucl. Phys. 60, 82 (2008).

[3] E. Epelbaum, H. W. Hammer and U.-G. Meißner, Rev. Mod. Phys. 81, 1773 (2009).

[4] N. Brambilla et al., Eur. Phys. J. C 71, 1534 (2011).

[5] N. Brambilla et al., Eur. Phys. J. C 74, no. 10, 2981 (2014).

[6] H. X. Chen, W. Chen, X. Liu and S. L. Zhu, Phys. Rept. 639, 1 (2016).

[7] F. K. Guo, C. Hanhart, U.-G. Meißner, Q. Wang, Q. Zhao and B. S. Zou, arXiv:1705.00141 [hep-ph].

[8] T. Aushev et al. [Belle Collaboration], Phys. Rev. D 81, 031103 (2010).

[9] A. Garmash et al. [Belle Collaboration], Phys. Rev. Lett. 116, 212001 (2016).

[10] A. E. Bondar, A. Garmash, A. I. Milstein, R. Mizuk and M. B. Voloshin, Phys. Rev. D 84054010 (2011).

[11] V. Baru, E. Epelbaum, A. A. Filin, C. Hanhart, U.-G. Meißner and A. V. Nefediev, Phys. Lett. B 763, 20 (2016).

[12] V. Baru, E. Epelbaum, A. A. Filin, C. Hanhart and A. V. Nefediev, JHEP 1706, 158 (2017).

[13] M. T. AlFiky, F. Gabbiani and A. A. Petrov, Phys. Lett. B 640, 238 (2006).

[14] T. Mehen and J. W. Powell, Phys. Rev. D 84114013 (2011).

[15] J. Nieves and M. P. Valderrama, Phys. Rev. D 86, 056004 (2012).

[16] ALPHA collaboration, F. Bernardoni, J. Bulava, M. Donnellan and R. Sommer, Phys. Lett. B 740, 278 (2015).

[17] V. Baru, A. A. Filin, C. Hanhart, Y. S. Kalashnikova, A. E. Kudryavtsev and A. V. Nefediev, Phys. Rev. D 84, 074029 (2011).

[18] V. Baru, E. Epelbaum, A. A. Filin, J. Gegelia and A. V. Nefediev, Phys. Rev. D 92, no. 11, 114016 (2015).

[19] V. Baru, E. Epelbaum, A. A. Filin, C. Hanhart, U.-G. Meißner and A. V. Nefediev, Phys. Lett. B 726, 537 (2013). 